\title{
Single molecule chromogenic in situ hybridization assay for RNA visualization in fixed cells and tissues
}

\author{
MENG JIANG, ${ }^{1}$ LING LIU, ${ }^{1}$ CHENGYE HONG, ${ }^{2}$ DEBO CHEN, ${ }^{2}$ XIHU YAO, ${ }^{2}$ XIAOYUAN CHEN, ${ }^{1}$ CHEN LIN, ${ }^{1}$ \\ and RONGQIN KE ${ }^{1}$ \\ ${ }^{1}$ Center for Precision Medicine, School of Biomedical Sciences and School of Medicine, Huaqiao University, Quanzhou, Fujian, 362021, China \\ ${ }^{2}$ Quanzhou First Hospital Affiliated to Fujian Medical University, Quanzhou, Fujian, 362000, China
}

\begin{abstract}
Visualization of gene expression at single RNA molecular level represents a great challenge to both imaging technologies and molecular engineering. Here we show a single molecule chromogenic in situ hybridization (smCISH) assay that enables counting and localizing individual RNA molecules in fixed cells and tissue under bright-field microscopy. Our method is based on in situ padlock probe assays directly using RNA as a ligation template and rolling circle amplification combined with enzyme catalyzed chromogenic reaction for amplification product visualization. We show potential applications of our method by detecting gene expression variations in single cells, subcellular localization information of expressed genes, and gene expression heterogeneity in formalin-fixed, paraffin-embedded tissue sections. This facile and straightforward method can in principle be applied to any type of RNA molecules in different samples.
\end{abstract}

Keywords: chromogenic in situ hybridization; padlock probe; rolling circle amplification; single molecule

\section{INTRODUCTION}

Owing to the recent advances in imaging technologies and molecular engineering, RNA can now be visualized in situ at the single molecule level, offering cellular and subcellular resolution (Crosetto et al. 2015). This highresolution spatiotemperal information can help us to better understand the physiological and pathological functions of detected genes. Currently, most of the strategies to achieve in situ single molecule RNA detection are based on generating elevated foreground fluorescence signals that can be distinguished from the background fluorescence, such as single molecule fluorescence in situ hybridization (smFISH) (Femino et al. 1998), methods based on rolling circle amplification (Larsson et al. 2010), methods based on hybridization chain reaction (HCR) (Shah et al. 2016a), and methods based on branched DNA (bDNA) technology (Battich et al. 2013). Combined with molecular barcoding strategies like in situ sequencing (Ke et al. 2013; Lee et al. 2014) and sequential hybridization (Chen et al. 2015; Shah et al. 2016b), these methods can be used to profile transcriptomics in situ, which can help to construct the potential regulatory network of gene expression (Lein et al. 2017). However, these fluorescence detection-based techniques all rely on detection

Corresponding authors: chen.lin@hqu.edu.cn, rke@hqu.edu.cn

Article is online at http://www.rnajournal.org/cgi/doi/10.1261/rna. 070599.119 probes labeled with organic dyes, which may suffer from a photobleaching-caused weak signal. In order to overcome the fluorescence deterioration problem of organic dyes, a recent study successfully utilized compact inorganic quantum dots labeled oligonucleotide as detection probes in smFISH for RNA in situ detection (Liu et al. 2018).

Although inorganic quantum dots as labels provide more stable signals over the traditional organic fluorescence dyes, autofluorescence occurs in certain samples and still remains a challenge for fluorescence-based single molecule RNA detection. Autofluorescence in tissue samples can arise from natural biomolecular components like lipofuscin and elastin or caused by fixation processes that generate fluorescence chemical groups in the pretreatment processes (Monici 2005). Strong autofluorescence leads to low signal to noise ratio that can even mask the true signal, resulting in detection failure. According to their sources, autofluorescence can be reduced either by chemical reagents, photobleaching, tissue clearing, or image processing approaches (Van de Lest et al. 1995; Clancy and Cauller 1998; Schnell et al. 1999; Neumann and Gabel 2002; Richardson and Lichtman 2015). However, an

(C) 2019 Jiang et al. This article is distributed exclusively by the RNA Society for the first 12 months after the full-issue publication date (see http://rnajournal.cshlp.org/site/misc/terms.xhtml). After 12 months, it is available under a Creative Commons License (Attribution-NonCommercial 4.0 International), as described at http:// creativecommons.org/licenses/by-nc/4.0/. 
alternative and simple way to avoid autofluorescence is to use chromogenic assays for signal development. Enzyme catalyzed chromogenic reactions have been widely used in conventional chromogenic in situ hybridization (CISH) protocols for both RNA and DNA detection (Volpi et al. 2018). Once the chromogenic signal is developed, it can be fixed and preserved for long-term storage by using mounting medium, which is of great advantage for tissue samples that need to be archived and reaccessed when needed. However, conventional methods do not offer RNA detection at single molecule resolution and sometime suffer from poor specificity and sensitivity. Currently, the bDNA-based RNAScope technology offers chromogenic assays for RNA in situ detection whose signal is discrete dots that can be quantified digitally (Wang et al. 2012). In order to perform this assay, multiple pairs of double "Z" probes need to be used to target the same RNA molecule, and several rounds of amplification probes have to be hybridized before the signal can be detected. Although this commercially available technique is attracting more and more users, its technical detail is not disclosed and the cost of such an assay is relatively high.

In this paper, we developed a simple and straightforward single molecule chromogenic in situ hybridization (smClSH) assay for sensitive and specific detection of RNA in fixed cells and tissues based on padlock probing and rolling circle amplification (Nilsson et al. 1994; Baner et al. 1998). In the original in situ padlock probe assay for in situ RNA detection, mRNA needs to be reverse transcribed into cDNA before it can be used as a padlock probe ligation template (Larsson et al. 2010). Recently, chlorella virus DNA ligase was shown to be able to ligate DNA probes on RNA template efficiently (Lohman et al. 2014). Taking advantage of this discovery, several studies have demonstrated that padlock probes can be directly ligated on RNA templates for in situ detection of single RNA molecules, simplifying the protocol by skipping the reverse transcription step (Deng et al. 2017; Schneider and Meier 2017). However, the fidelity of splintR DNA ligase does not seem to be poor, as shown by a study that it can tolerate mismatches in the ligation junction (Krzywkowski and Nilsson 2017). Therefore, the success of RNA template-initiated padlock probe assay for in situ RNA detection needs to depend on the specificity of hybridization. According to these findings, we designed a padlock probing protocol that can generate specific and sensitive in situ RNA detection results for our smFISH methillustrated in $A$. od, which is shown in Figure 1. First, padlock probes are hybridized to their gene-specific target sequence on the mRNA molecules. Allowing for efficient hybridization under stringent conditions, the excess amount of the probes is then washed away. The ligation of hybridized padlock probes is then performed as a separate step, generating circularized DNA molecules that serve as RCA templates. In order to make sure that the RCA can be initiated efficiently, an RCA primer is hybridized to the circularized padlock probe on its backbone sequence. After removal of the nonhybridized primers, RCA is initiated to generate rolling circle amplification products (RCPs). RCPs are then hybridized with HRP labeled oligonucleotide probes, followed by enzyme catalyzed color development to visualize individual RCPs that corresponds to individual RNA molecules. We used 3,3'-Diaminobenzidine (DAB)-based substrate for HRP to stain the RCPs and hematoxylin to stain the cell nuclei, generating RCP dots in brown color and nuclei in blue color. We can quantify the RCPs per cell by the open-source software CellProfiler by compiling a pipeline using its builtin modules (Carpenter et al. 2006). We demonstrated that our method is feasible by comparing the detection results to fluorescence detection and then used this method to distinguish gene expression variations in different cell types as well as gene expression alteration before and after drug stimulation. We also demonstrated that our method can be applied to clinical samples by successful detection of HER2 expression status in routine formalin-fixed, paraffinembedded (FFPE) pathological tissue sections. Our results show that single RNA molecules can be detected by chromogenic assay in situ, providing an alternative approach to detect in situ gene expression to avoid autofluorescence in

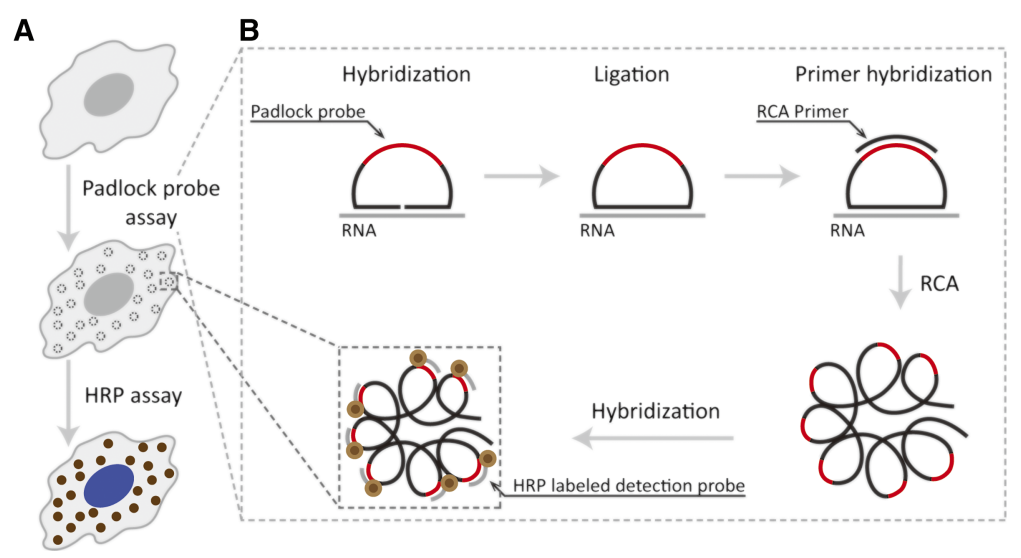

FIGURE 1. The principle of smCISH. (A) Overview of the smFISH for detection of individual RNA in situ. (B) Detailed molecular procedures of padlock probe assay for smCISH: Genespecific padlock probe is hybridized directly to its RNA target. Next, probe circularization is achieved by RNA-templated ligation of its two ends. An RCA primer is then hybridized to the circularized padlock probe, followed by performing rolling circle amplification to generate RCP, thus enabling signal amplification. RCP is hybridized with HRP labeled detection probes. Finally, DAB staining is used to visualize the RCP and nucleus is stained blue by hematoxylin, as 
certain samples with a stable signal. Furthermore, the method can in principle be applied to any type of RNA molecules in various samples.

\section{RESULTS AND DISCUSSION}

\section{Optimization of padlock probe and RCA-based smCISH}

In order to establish a protocol that is versatile, we performed optimization experiments to tweak the experimental conditions. First, we compared the padlock probe hybridization time by performing detection of HER2 expression in the SK-BR-3 cell line. The hybridization reactions were allowed to stay for $30 \mathrm{~min}, 2 \mathrm{~h}$, and $4 \mathrm{~h}$, followed by performing the same protocols afterwards (Fig. 2A-C). RCPs were counted and compared as shown in Figure 2D. The results show that the number of counted RCPs increased by the extension of hybridization time. We chose $2 \mathrm{~h}$ as the hybridization time because the increase of signal from $2 \mathrm{~h}$ to $4 \mathrm{~h}$ hybridization is not as obvious as that from 30 min to $2 \mathrm{~h}$, thus also allowing the protocol to be continued within a shorter waiting time.

To investigate if the hybridization efficiency of HRP labeled detection probes can be improved, different concentrations of glycerol were added to the hybridization mix. The data showed that $20 \%$ of glycerol resulted in the highest RCP number per cell so that this concentration was used in the following experiments (Supplemental Fig. S1). In order to simplify the protocol, we tried to integrate the RCA primer hybridization with RCA by adding the RCA

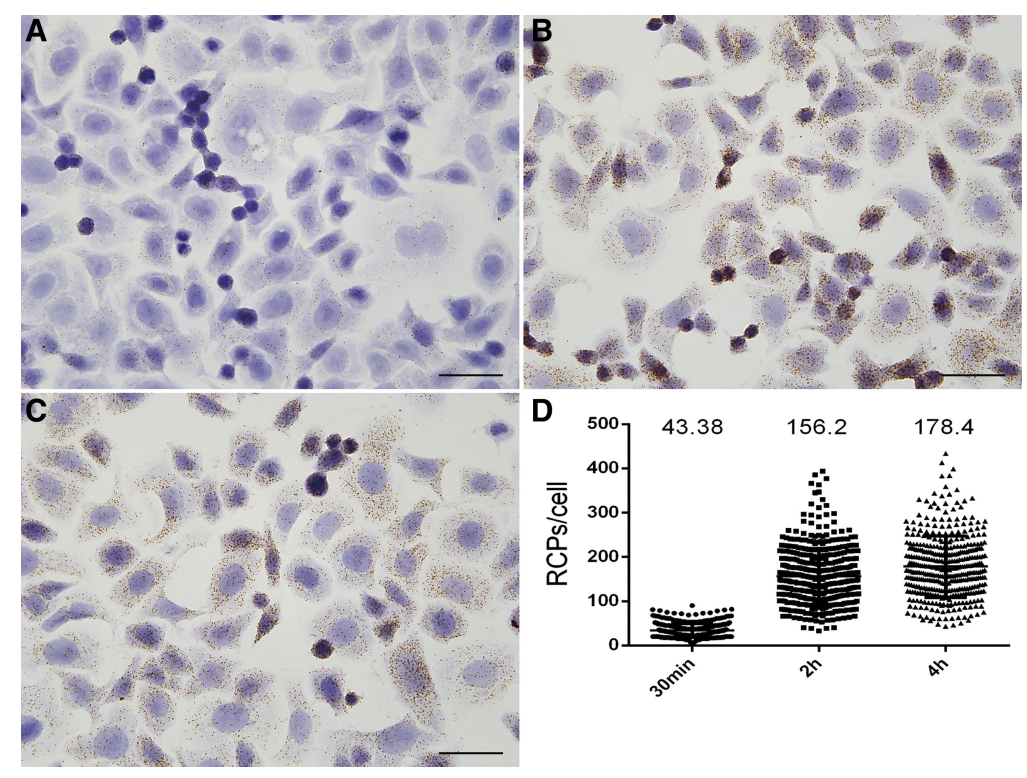

FIGURE 2. Effects of different padlock probe hybridization time on detecting efficiency. Padlock probes were hybridized with HER2 mRNA in SK-BR-3 for 30 min (A), $2 \mathrm{~h}(B)$, and $4 \mathrm{~h}$ (C). (D) Quantification of HER2 mRNA detection with different probe hybridization times: 30 $\min (n=370), 2 \mathrm{~h}(n=438)$, and $4 \mathrm{~h}(n=399)$. Scale bar, $40 \mu \mathrm{m}$. primers into the RCA reaction mix to perform the primer hybridization and RCA in the same step. However, the results showed that separating the RCA primer hybridization from the RCA step generates more RCPs (Supplemental Fig. S2), indicating that the primer hybridization is not efficient when combined with RCA. Therefore, the primer hybridization was kept as a separate step.

\section{Comparison of smCISH with fluorescence detection}

We then used our method to detect ACTB mRNA expression in the breast cancer cell line SK-BR-3. As shown in Figure 3A, individual ACTB mRNA molecules are visualized as discrete brown dots in single SK-BR-3 cells whose nuclei are shown in blue. We side-by-side compared our method to that of conventional fluorescence probe detection by performing the same procedure, except that the RCPs were visualized with fluorophore labeled in the fluorescence detection (Fig. 3B). Both protocols resulted in very similar round shaped and discrete dots for RCPs, and the detection efficiency seems to be very similar, according to CellProfiler analysis, with 142.6 \pm 70.05 RCPs per cell $(n=287)$ for chromogenic detection and $135.7 \pm$ 71.75 RCPs per cell $(n=345)$ for fluorescent detection. Therefore, there are no statistical differences between these two methods $(P<0.05)$. We observed a signal overcrowding problem both in the chromogenic assay and fluorescence assay in the cells that have a high ACTB expression level. The overcrowding problem is similar to that discussed in a previous study (Ke et al. 2013). We also compared detection of GAPDH in a fresh frozen mouse brain tissue, which we previously found to have a strong autofluorescence. When detected with smCISH, we were able to visualize and locate the individual GAPDH dots much easier than that of using fluorescent detection, where the strong autofluorescence makes it difficult to distinguish the signal from the background (Fig. 3C,D).

\section{Detection of gene expression variation by $\mathrm{smClSH}$}

In order to test if our method can detect gene expression variations, we performed smCISH for detection of HER2 mRNA in three cell lines (Fig. 4A-C) whose HER2 mRNA expression levels are low, moderate, and high according to the Human Protein Atlas database (https://www .proteinatlas.org). Our results showed that the expression level of HER2 


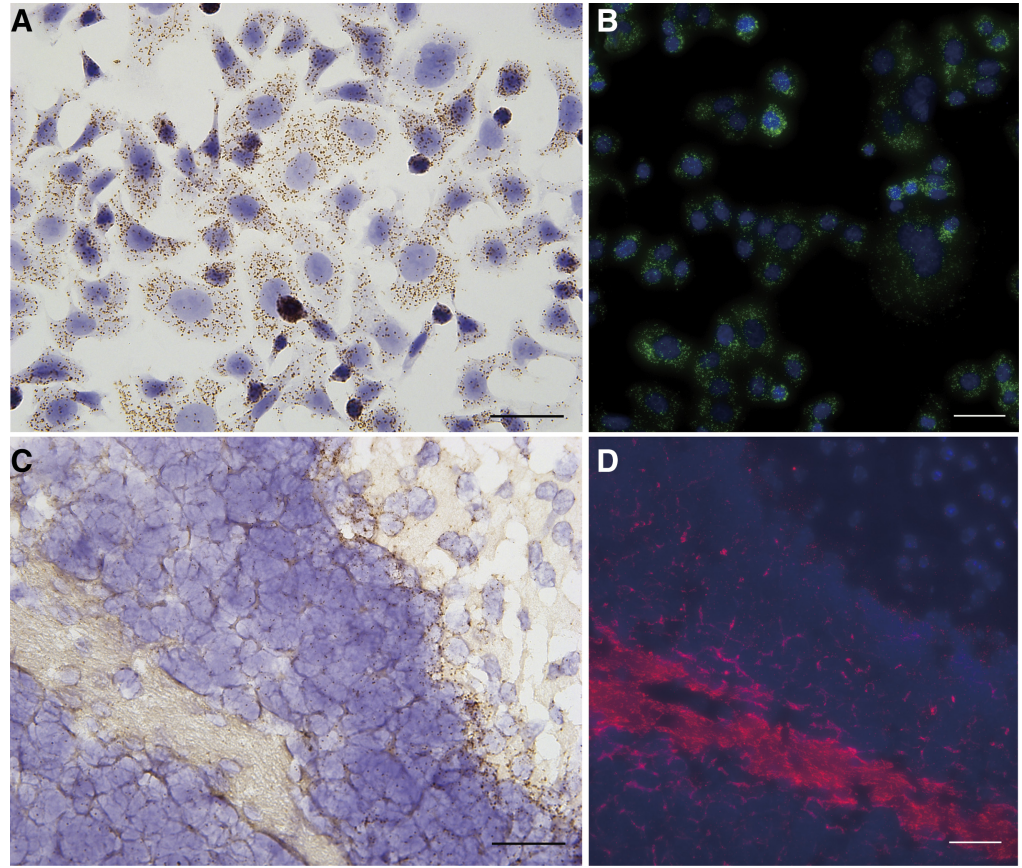

FIGURE 3. Comparison of RCA-based single molecule chromogenic and fluorescence in situ hybridization assay for individual ACTB mRNA detection in SK-BR-3 cells $(A, B)$ and GAPDH mRNA detection in fresh frozen mouse brain tissues $(C, D)$. For chromogenic detection $(A, C)$, brown dots were detected. mRNA RCPs stained with DAB and nuclei were counterstained blue with hematoxylin. Scale bar, $40 \mu \mathrm{m}$. For fluorescent detection $(B, D)$, green dots $(A C T B)$ and red dots (GAPDH) were detected. mRNA RCPs and the nuclei were counterstained blue with DAPI. Scale bar, $40 \mu \mathrm{m}$.

mRNA is highest in SK-BR-3 cells and lowest in MCF-7 cells, along with an expression level in T47D cells in between (Fig. 4D). This result was consistent with the TPM values shown in the Human Protein Atlas database, showing the specificity of our smClSH assay. Although the MCF-7 cell line was classified as HER2-negative by immunohistochemistry in some studies, other studies also showed that there is a low level of HER2 expression, which agrees with the HER2 mRNA expression level in the HPA database (Emde et al. 2011). As mentioned above, the RNA template-initiated padlock probe ligation and circularization using splintR DNA ligase may suffer from poor fidelity, making the specificity of our smCISH assay mainly dependent on the hybridization step. One major concern of using this approach is that the specificity can be poor due to nonspecific hybridization and ligation. Our data show that the specificity of our method is reliable, because the different expression levels of the same gene in different cell lines can be clearly distinguished. Although the other studies investigate the specificity of their assays by discriminating a single nucleotide mismatch at the end of the padlock probes (Deng et al. 2017; Schneider and Meier 2017), the fidelity study of the SplintR DNA ligase has already shown that discrimination ability is poor and depends very much on the bases being ligated (Krzywkowski and Nilsson 2017). Moreover, in most applications of in situ
RNA detection, discriminating single nucleotide polymorphism is not necessary. Therefore, this kind of specificity study does not reflect the specificity of the whole assay. Herein, we show that different expression levels of the same gene in different cell lines originating from the same type of cancer can be distinguished by our assay, indicating that our method can detect target RNA molecules specifically.

To investigate if our smCISH assay can be used for distinguishing the RNA expression level change after stimulation of cells, we detected mRNA expression levels of PD-L1, also known as CD274, in the H1299 cell line (Supplemental Fig. S3). It is known that the up-regulation of PDL1 helps the cancer to evade the host immune system. The expression level of PD-L1 can increase by the IFN- $\gamma$ simulation. Our data show that after $24 \mathrm{~h}$ of IFN- $\gamma$ simulation, the $\mathrm{H} 1299$ cells expressed much higher levels of PD-L1 than the control cells without stimulation. This result indicates that gene expression level change can be digitally quantified by our smCISH assay. This kind of digital quantification is more straightforward than real-time quantitative PCR that compares the $C_{t}$ values.

\section{Specificity and detection efficiency of smCISH}

To further investigate the specificity of $\mathrm{smClSH}$, we performed detection of enhanced green fluorescence protein gene (EGFP) mRNA expression in the CA46 cells infected with lentivirus vectors carrying an EGFP reporter gene and noninfected CA46 cells as negative control. The average RCPs detected per cell is 4.681 versus 0.06604 in the infected cells and the negative control respectively, indicating that only a very few nonspecific signal dots were detected (Supplemental Fig. S4). The result shows that smClSH can detect the mRNA expression from a heterologous gene very specifically, further proving the specificity of our smClSH assay.

To investigate the detection efficiency of smClSH, we compared our assay to the original padlock probe and RCA-based in situ RNA detection assay that used cDNA as ligation template (Supplemental Fig. S5; Supplemental Table S1). The number of detected HER2 transcripts in A549 and MCF-7 cells using RNA as a ligation template was around twofold and threefold of that when using cDNA as ligation template, showing increased detection 


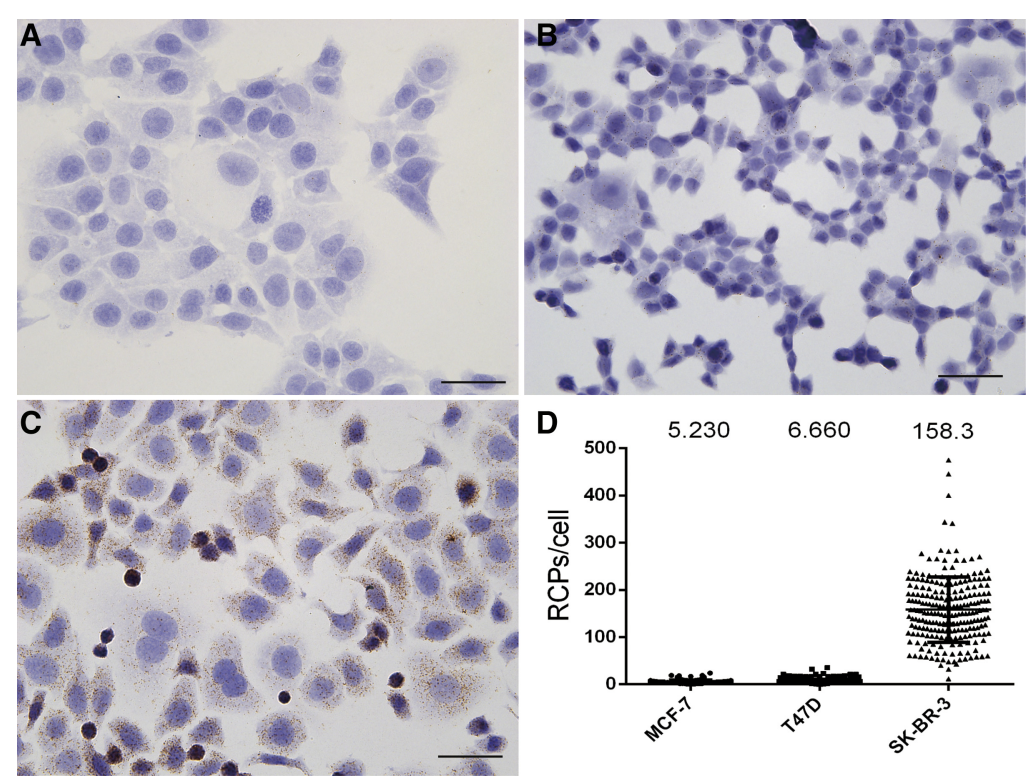

FIGURE 4. HER2 mRNA expression levels in different breast cancer cell lines. (A) HER2 mRNA was detected as low expression in MCF-7. (B) HER2 mRNA was moderately expressed in T47D. (C) HER2 mRNA was strongly expressed in SK-BR-3. (D) Quantification of HER2 mRNA detection in MCF-7 $(n=239)$, T47D $(n=371)$, and SK-BR-3 $(n=228)$. Scale bar, $40 \mu \mathrm{m}$.

RNA molecule on different sites should be used when detecting a new gene.

\section{Visualization of subcellular location of long noncoding RNA molecules}

We performed detection of long noncoding RNA (IncRNA) MALAT1 and CYTOR in cultured cancer cells H1299 and SW480 to test if we can detect the subcellular location of RNA molecules. MALAT1 and CYTOR are previously known to be mainly expressed in the nucleus and cytoplasm (Soares et al. 2017). As shown in Figure 5 , we can see that most of the MALAT1 are detected in the nuclei and there are more CYTOR located in the cytoplasm than that in the nuclei. However, since the expression level analysis was based on two-dimensional images, the CYTOR detected in the nuclei

efficiency of our current protocol (Supplemental Fig. S5; Supplemental Table S1). When we compared our results to those of smFISH and rollFISH in Wu et al. (2018), the detection efficiency of smCISH that used only one padlock probe was much lower than that of smFISH and rollFISH (Supplemental Table S1). However, when we increased the number of padlock probes to three, the RCPs that could be detected per cell also increased. Although the median detected HER2 signal spots in A549 for smCISH was around half of that of the rollFISH and smFISH (12 per cell for smCISH versus 21 per cell for rollFISH and 26 per cell for smFISH), we detected more HER2 signals in the MCF-7 cells (rollFISH detected more HER2 signals in A549 than in MCF-7 in the referenced paper [Wu et al. 2018]). Considering that we were not using exactly the same cells as in the reference, we can conclude that our smClSH can achieve comparable detection efficiency as smFISH and rollFISH when using multiple padlock probes. The reason is that when there is inefficient binding of padlock probes, steric hindrance of the probe binding site, or potential fragmentation of RNA in the probe binding site, only using one padlock probe can result in miss of the target, but multiple padlock probes have a higher chance of finding the right targets and generate a detectable signal. Although our results showed that the RNA as ligation template strategy had higher detection efficiency than the original cDNA as a ligation template approach, the detection efficiency was still not as good as that of smFISH when only one probe was used. Therefore, in order to achieve better detection efficiency and to avoid false negative results, at least three padlock probes that target the same can actually be located above the nuclei. Therefore, such a type of subcellular localization detection only provides superficial localization information. Nevertheless, the successful detection of MALAT1 indicates that our smCISH can be used to detect RNA expressed in the nuclei that has a higher density of biomolecules.

\section{In situ detection of HER2 mRNA expression in clinical tissue sections by $\mathrm{smClSH}$}

The previous data shows the smCISH assay can be used to specifically detect individual mRNA molecules in cell culture. We wanted to see if our method can also work in routine clinical samples, that is, FFPE tissue sections. An ideal method for in situ RNA detection should be able to handle FFPE samples. We performed in situ HER2 mRNA detection on two slides that were previously classified as HER2-positive and HER2-negative by immunohistochemistry in the diagnostics laboratory. The smCISH result is showed in Figure 5. In the HER2-positive tissue section, we can observe obvious brown color staining patterns that consist of many brown RCP dots in the cancer cells (Fig. 6A). We can also notice that there is no signal in the normal cell area, demonstrating the HER2 expression heterogeneity in this tissue section together with the previous observation in the cancer cells. In the HER2-negative tissue section, we only saw very few RCP dots across the section (Fig. 6B). Although this agrees with the immunohistochemistry results, further developments still need to be conducted before our method can be used in clinical settings. For example, an expression level grading system needs to be 
A

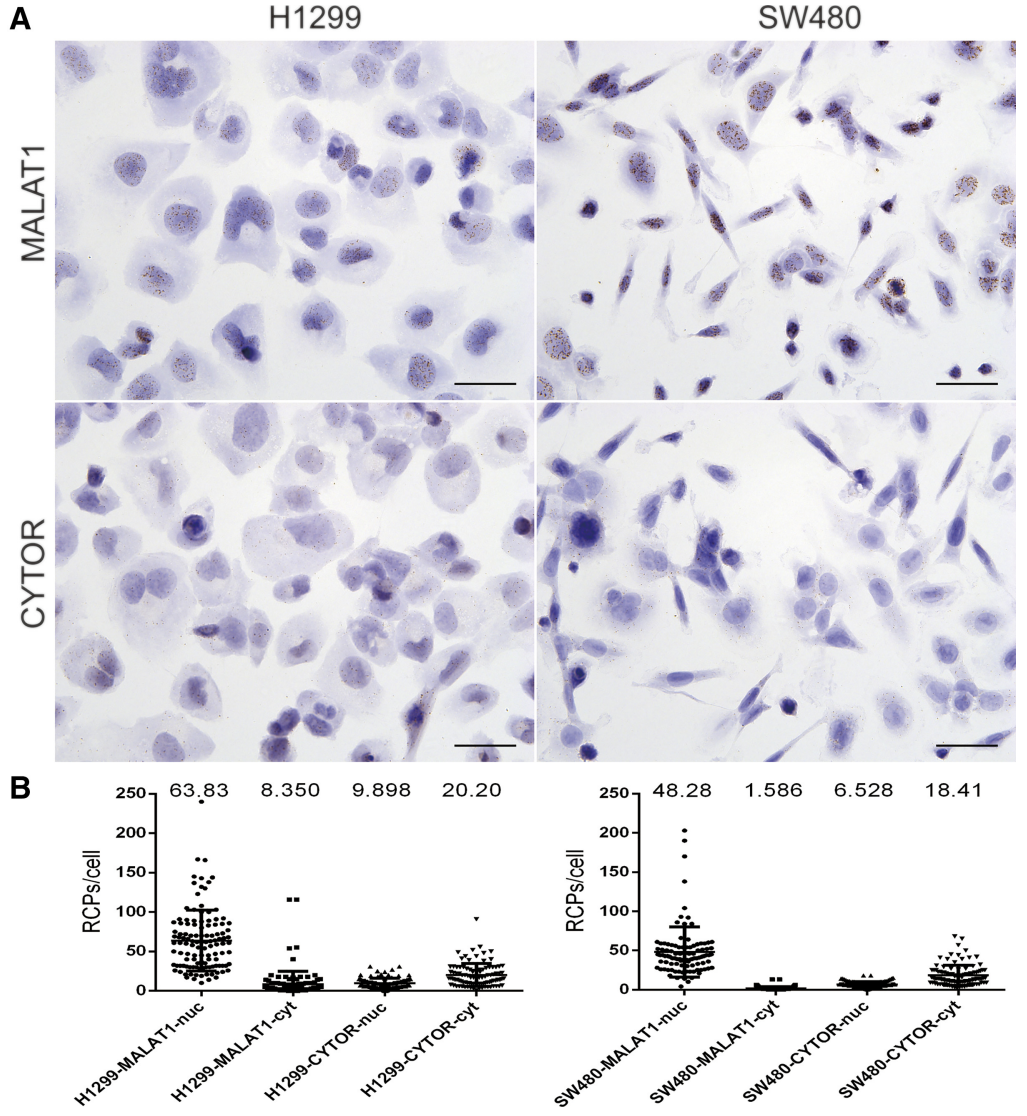

FIGURE 5. Visualization of subcellular location of long noncoding RNA molecules. (A) MALAT1 and CYTOR RNA molecules are detected as brown dots in fixed H1299 and SW480 cells. The nuclei are stained in blue. Scale bar, $40 \mu \mathrm{m}$. (B) Comparison of the expression levels of MALAT1 (H1299: $n=117$, SW480: $n=99)$ and CYTOR $(H 1299: n=98$, SW480: $n=$ $106)$ in the nuclei and the cytoplasm.

established, which could be similar to that used in the commercially available RNAScope method, in which they define the grades of expression by counting signal dots per cell. Nonetheless, our data show that our smClSH assay can be applied to clinical tissue samples for RNA in situ detection.

\section{Conclusion}

Today, outsourcing molecular diagnostics to wellequipped centralized laboratories is becoming a trend. In this paper, on the contrary, we developed a method using chromogenic assay for single molecule RNA in situ detection, making it possible to gain "high-end" results by a "low-tech" approach. The method does not depend on delicate or advanced devices, therefore it can be used in less well-equipped laboratories and potentially be further developed into a point-of-care method for disease diagnosis. We have achieved visualization of individual RNA molecules in situ using bright-field microscopy that provides cellular and subcellular resolution. The method not only works in fixed cells, but more importantly also works well in FFPE tissue sections. However, in order to increase the detection efficien$c y$, three or more padlock probes should be used to avoid false negatives caused by probes failing to ligate on their targets. Although the ability to multiplex is quite limited, the simplicity of this method still makes it easy to adapt. We believe this method will find its place in both basic life science research and potentially in clinical diagnostics.

\section{MATERIALS AND METHODS}

\section{Cell culture and sample preparation}

The cell lines SK-BR-3, H1299, SW480, and CA46 were cultured in RPMI 1640 (Thermo Scientific), supplemented with $10 \%$ fetal bovine serum (FBS, Thermo Scientific) and $1 \%$ Penicillin-Streptomycin Solution (Thermo Scientific). The cell line SK-OV-3 was cultured in McCoy's 5A Medium (Thermo Scientific), supplemented with $10 \%$ FBS and 1\% Penicillin-Streptomycin Solution (Thermo Scientific). The cell lines MCF-7, A549, and T47D were cultured in DMEM (Thermo Scientific), supplemented with $10 \%$ FBS and $1 \%$ Penicillin-Streptomycin Solution. All cell lines were incubated at $37^{\circ} \mathrm{C}, 5 \% \mathrm{CO}_{2}$.

To prepare cell samples, cells were treated with $0.25 \%(\mathrm{w} / \mathrm{v})$ trypsin-EDTA (Thermo Scientific) when confluent and resuspended in culturing medium. Resuspended cells were then evenly seeded on the Superfrost Plus slides (Thermo Scientific) submerged in culturing medium placed in a $150 \mathrm{~mm} \times 25 \mathrm{~mm}$ Petri dish (Corning). The culturing medium was added to a final volume of $25 \mathrm{~mL}$. Cells were allowed to grow under the same conditions for 12 to $48 \mathrm{~h}$ before fixation. After removal of the culturing medium and two washes with diethyl pyrocarbonate (DEPC) treated PBS (DEPC-PBS), fixation was carried out by incubating the slides in $4 \%(w / v)$ PFA in DEPC-PBS for 30 min at room temperature (RT). Slides were then washed with DEPC-PBS twice and dehydration of the slides was performed using an ethanol series of $70 \%$, $85 \%$, and ethanol absolute for 5 min each. These slides can then be stored at $-80^{\circ} \mathrm{C}$ until used.

For CA46 cell samples, cells were collected by centrifuging at $1500 \mathrm{rpm}$ for $5 \mathrm{~min}$. After removal of the culturing medium and two washes with DEPC-PBS, fixation was carried out by adding $4 \%(\mathrm{w} / \mathrm{v})$ PFA in DEPC-PBS and incubated $30 \mathrm{~min}$ at RT directly in the tube. After fixation, cells were washed with DEPC-PBS once, followed by resuspension of the cell in the same buffer. Resuspended cells in DEPC-PBS were then seeded on the PolyL-Lysine-Prep slides (Sangon) by direct pipetting the cell 

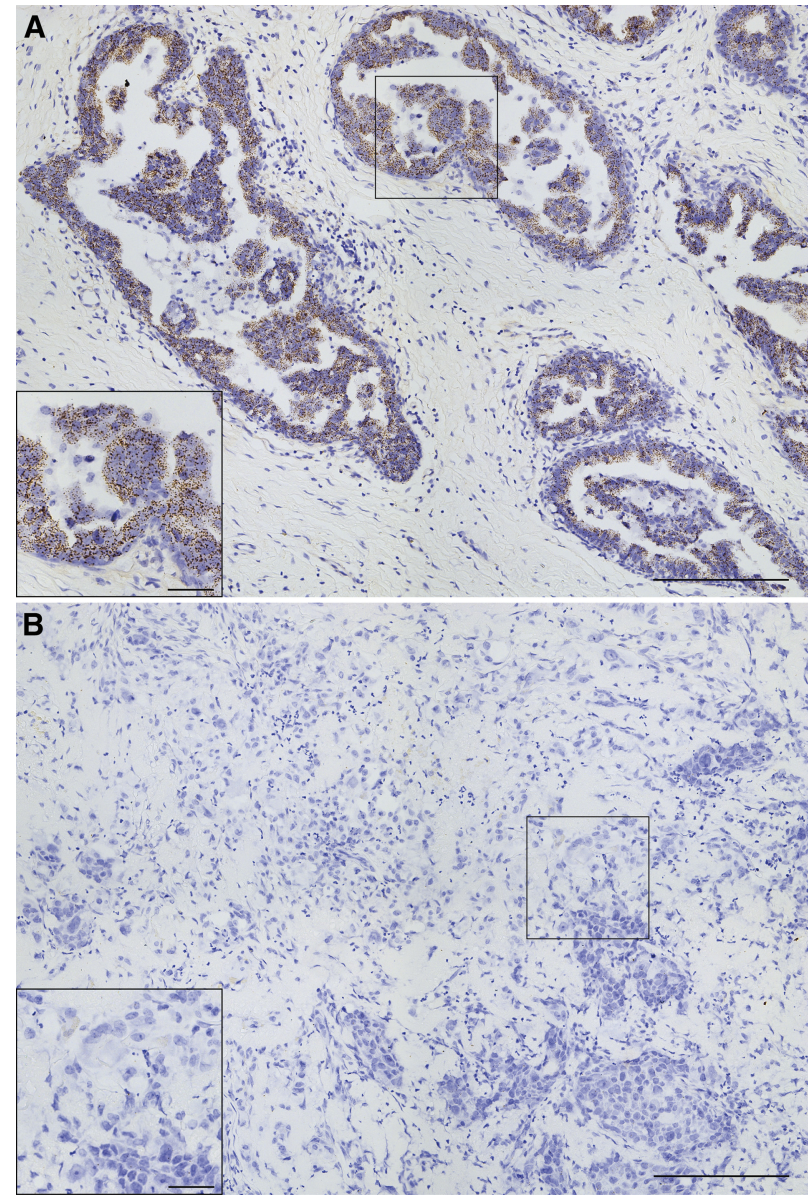

FIGURE 6. Detection of HER2 mRNA expression in breast cancer FFPE tissue sections. (A) HER2-positive and (B) HER2-negative FFPE breast cancer tissue sections. Scale bar, $200 \mu \mathrm{m}$ for main image and $40 \mu \mathrm{m}$ for insert.

suspension on the slide surface and then air-dried. These slides can then be stored at $-80^{\circ} \mathrm{C}$ until used.

\section{Pretreatments}

Cell samples were first rinsed with DEPC-PBS with $0.05 \%$ Tween 20 (Sigma) (DEPC-PBST) and then permeabilized by incubating with $0.5 \%(\mathrm{v} / \mathrm{v})$ Triton X-100 (Sigma) (in DEPC-PBS) for $10 \mathrm{~min}$. For FFPE tissue sections, slides were first baked at $60^{\circ} \mathrm{C}$ for $30 \mathrm{~min}$, then deparaffinized by exposure to xylene twice for $15 \mathrm{~min}$ and $10 \mathrm{~min}$, respectively, followed by submerging in ethanol absolute twice, 95\% ethanol twice, and $70 \%$ ethanol twice for 2 min each time. To complete rehydration, slides were treated with DEPC- $\mathrm{H}_{2} \mathrm{O}$ for $5 \mathrm{~min}$, then washed with DEPC-PBS for $2 \mathrm{~min}$. Then, fixation was performed in 4\% (w/v) PFA (Sigma) in DEPCPBS for $10 \mathrm{~min}$. A solution of $0.1 \mathrm{mg} / \mathrm{mL}$ pepsin (Sigma) in 0.1 $\mathrm{M} \mathrm{HCl}$ was applied to the slides for $30 \mathrm{~min}$ at $37^{\circ} \mathrm{C}$ after one wash in DEPC-PBS for $2 \mathrm{~min}$. Next, slides were treated with DEPC- $\mathrm{H}_{2} \mathrm{O}$ for $5 \mathrm{~min}$ and DEPC-PBS for $2 \mathrm{~min}$, followed by dehydration with an ethanol series of $70 \%, 85 \%$ and ethanol absolute for $1 \mathrm{~min}$ each. For fresh frozen mouse tissue sections, slides were first fixed in 4\% (w/v) PFA in DEPC-PBS for 5 min. After two washes with DEPC-PBS for 2 min each time, the slides were dipped into an ethanol serial of $70 \%, 85 \%$ and ethanol absolute for $1 \mathrm{~min}$ each. Then a solution of $1 \mathrm{mg} / \mathrm{mL}$ pepsin in $0.1 \mathrm{M} \mathrm{HCl}$ was applied to the slides for $5 \mathrm{~min}$ at $37^{\circ} \mathrm{C}$, followed by three washes with DEPC-PBST.

From here on, all of the following reactions were performed in $9 \mathrm{~mm}$ diameter and $0.8 \mathrm{~mm}$ depth Secure-Seal hybridization chambers (Thermo Scientific) attached to the surface of the slide on the side where the samples were, unless otherwise stated.

\section{Padlock probe hybridization}

The sample was first washed with DEPC-PBST three times, then a $3 \%(\mathrm{w} / \mathrm{v}) \mathrm{H}_{2} \mathrm{O}_{2}$ solution was applied to the sample and allowed to incubate for $15 \mathrm{~min}$ to complete the blocking of intrinsic peroxidase, followed by another three washes with DEPC-PBST. Next, $100 \mathrm{nM}$ padlock probe (all probe sequences are listed in Supplemental Table S2) in hybridization buffer (10\% formamide in $6 \times$ SSC buffer) was added to the sample and incubated for $2 \mathrm{~h}$ or as stated at $37^{\circ} \mathrm{C}$, followed by three washes for 5 min each with washing buffer containing $2 \times$ SSC and $20 \%$ formamide to remove excess unhybridized padlock probes. Then the sample was washed three times with DEPC-PBST to get rid of formamide, which can potentially affect the activity of the enzymes used in the following step.

\section{Padlock probe ligation}

A ligation mix containing $1 \times$ SplintR ligase reaction buffer (NEB), $50 \%$ glycerol, $0.5 \mathrm{U} / \mu \mathrm{L}$ SplintR ligase (NEB), $1 \mathrm{U} / \mu \mathrm{L}$ RiboLock RNase Inhibitor (Thermo Scientific), $0.2 \mu \mathrm{g} / \mu \mathrm{L}$ BSA (NEB) in DEPC $-\mathrm{H}_{2} \mathrm{O}$ was added to the sample and allowed to incubate for $1 \mathrm{~h}$ at $37^{\circ} \mathrm{C}$, followed by three rinses with washing buffer for $5 \mathrm{~min}$ each to remove the unligated padlock probes and enzyme.

\section{Original cDNA as ligation template approach}

For the method of in situ RNA detection assay that used cDNA as ligation template, mRNA was copied to cDNA by reverse transcription, followed by degradation of the mRNA strand. Then the padlock probe hybridization and ligation was carried out. The reversed transcription mix, containing $1 \mathrm{mM} d N T P s, 1 \mu \mathrm{M}$ LNA-modified reverse transcription primer, 20\% glycerol, 20 $\mathrm{U} / \mu \mathrm{L}$ RevertAid H Minus Reverse Transcriptase (Thermo Scientific), $1 \times$ reaction buffer for RevertAid H Minus Reverse Transcriptase (Thermo Scientific), $1 \mathrm{U} / \mu \mathrm{L}$ RiboLock RNase Inhibitor and $0.2 \mu \mathrm{g} / \mu \mathrm{L}$ BSA in DEPC- $\mathrm{H}_{2} \mathrm{O}$, was added to the samples and allowed to incubate for $3 \mathrm{~h}$ at $45^{\circ} \mathrm{C}$, followed by three washes with DEPC-PBST. Then, 4\% (w/v) PFA in DEPC-PBS was added to perform postfixation step for $30 \mathrm{~min}$ at RT. After postfixation, the samples were washed three times in DEPC-PBST. Padlock probe hybridization and ligation mix, containing $100 \mathrm{nM}$ padlock probe, $50 \mathrm{mM} \mathrm{KCl}, 20 \%$ formamide, $1 \times$ Ampligase DNA Ligase buffer, $0.5 \mathrm{U} / \mu \mathrm{L}$ Ampligase DNA Ligase, $0.4 \mathrm{U} / \mu \mathrm{L}$ RNase $\mathrm{H}$, $1 \mathrm{U} / \mu \mathrm{L}$ RiboLock RNase Inhibitor, and $0.2 \mu \mathrm{g} / \mu \mathrm{L}$ BSA in DEPC$\mathrm{H}_{2} \mathrm{O}$, was added to the samples and allowed to incubate for $30 \mathrm{~min}$ at $37^{\circ} \mathrm{C}$ for mRNA digestion, $45 \mathrm{~min}$ at $45^{\circ} \mathrm{C}$ for padlock 
probe hybridization and ligation. The samples were then washed three times by washing buffer.

\section{RCA primer hybridization}

One micromolar of RCA primer (primer sequences can be found in Supplemental Table S2) in hybridization buffer was added to the sample and incubated for $30 \mathrm{~min}$ at $37^{\circ} \mathrm{C}$, followed by three washes for 5 min each with washing buffer to remove unhybridized RCA primer. Then, three washes with DEPC-PBST were performed to prepare the samples for RCA.

\section{RCA}

An RCA mix containing $1 \times$ phi29 DNA polymerase buffer (Thermo Scientific), 5\% glycerol, $1 \mathrm{mM}$ dNTPs (Thermo Scientific), $1 \mathrm{U} / \mu \mathrm{L}$ phi29 polymerase (Thermo Scientific), $1 \mathrm{U} / \mu \mathrm{L}$ RiboLock RNase Inhibitor, $0.2 \mu \mathrm{g} / \mu \mathrm{L}$ BSA in DEPC- $\mathrm{H}_{2} \mathrm{O}$ was added to the sample and incubated for 16 to $18 \mathrm{~h}$ at RT to carry out RCA. After the incubation, the sample was rinsed three times with washing buffer for 5 min each. Then three washes with DEPC-PBST were performed to avoid the possible negative effect of formamide on the activity of HRP labeled on the detection probes.

\section{RCA products detection}

A detection mix containing 100 nM HRP labeled detection probes (Supplemental Table S2), 20\% glycerol (or other concentrations as stated), $2 \times$ SSC, $0.05 \%$ (v/v) Tween 20, and $0.4 \mu \mathrm{g} / \mu \mathrm{L}$ BSA in DEPC- $\mathrm{H}_{2} \mathrm{O}$ was added to the sample and incubated for $30 \mathrm{~min}$ at RT. Excess amounts of detection probes were removed by three washes with DEPC-PBST. For fluorescence in situ hybridization assay, a detection mix containing $100 \mathrm{nM}$ FAM labeled detection probes, 2x SSC, 20\% formamide in DEPC-H2O was added to the sample and incubated for $30 \mathrm{~min}$ at RT. Then, the slide was dipped into an ethanol serial of $70 \%$, $85 \%$ and ethanol absolute for 2 min each. The slides were mounted with SlowFade Gold Antifade Mountant (Thermo Scientific) containing $0.5 \mu \mathrm{g} / \mathrm{mL}$ DAPI (Sigma).

\section{Staining and mounting}

The sample was incubated with DAB staining mix (Sangon) containing $90 \%$ DAB buffer, 5\% DAB substrate solution (20x), and $5 \%$ DAB chromogen solution (20x) for 3 to 8 min depending on the color development before an immediate wash with water. Hematoxylin (Sigma) counterstaining was performed to visualize nucleic structures. After dehydration by an ethanol serial of $70 \%, 85 \%$ and ethanol absolute for $1 \mathrm{~min}$ each, the slides were mounted with neutral balsam mounting medium (Sangon).

\section{Image acquisition and analysis}

Images for all bright-field experiments were acquired with a Leica DM6B microscope equipped with a DFC7000T camera using the $40 \times$ objective except for the FFPE breast cancer tissue sections. The images for FFPE tissue sections were generated by stitching four images $(2 \times 2)$ obtained by using a $20 \times$ objective. The fluorescence images were obtained with a DFC9000GT camera using the $40 \times$ objective. The images were analyzed using CellProfiler image analysis software. The images were split into hematoxylin and DAB two channels with the "Unmixcolors" module. Then the background was removed and the DAB staining spots were highlighted with the "EnhanceOrSuppressFeatures" module. Finally, the relation of signals with their host cells was identified with the "RelateObjects" module. The number of RCPs per cell was compared across groups. Statistical analyses and plotting were performed using GraphPad Prism.

\section{SUPPLEMENTAL MATERIAL}

Supplemental material is available for this article.

\section{ACKNOWLEDGMENTS}

This work was supported by the funds from the National Key Research and Development Program of China (2017YFA01 06800), the National Natural Science Foundation of China (31770927), the Natural Science Foundation of Fujian Province (2019J01072), Subsidized Project for Postgraduate's Innovative Fund in Scientific Research of Huaqiao University, and the Scientific Research Funds of Huaqiao University (600005Z16Y0008). The authors would like to thank Dr. Tingjin Zheng for helping with the lentivirus infected cell line experiments.

Received January 29, 2019; accepted May 6, 2019.

\section{REFERENCES}

Baner J, Nilsson M, Mendel-Hartvig M, Landegren U. 1998. Signal amplification of padlock probes by rolling circle replication. Nucleic Acids Res 26: 5073-5078. doi:10.1093/nar/26.22.5073

Battich N, Stoeger T, Pelkmans L. 2013. Image-based transcriptomics in thousands of single human cells at single-molecule resolution. Nat Methods 10: 1127-1133. doi:10.1038/nmeth.2657

Carpenter AE, Jones TR, Lamprecht MR, Clarke C, Kang IH, Friman O, Guertin DA, Chang JH, Lindquist RA, Moffat J, et al. 2006. CellProfiler: image analysis software for identifying and quantifying cell phenotypes. Genome Biol 7: R100. doi:10.1186/gb2006-7-10-r100

Chen KH, Boettiger AN, Moffitt JR, Wang S, Zhuang X. 2015. Spatially resolved, highly multiplexed RNA profiling in single cells. Science 348: aaa6090. doi:10.1126/science.aaa6090

Clancy B, Cauller LJ. 1998. Reduction of background autofluorescence in brain sections following immersion in sodium borohydride. J Neurosci Methods 83: 97-102. doi:10.1016/S0165-0270 (98)00066-1

Crosetto N, Bienko M, van Oudenaarden A. 2015. Spatially resolved transcriptomics and beyond. Nat Rev Genet 16: 57-66. doi:10 $.1038 / \mathrm{nrg} 3832$

Deng R, Zhang K, Sun Y, Ren X, Li J. 2017. Highly specific imaging of mRNA in single cells by target RNA-initiated rolling circle amplification. Chem Sci 8: 3668-3675. doi:10.1039/C7SC00292K

Emde A, Mahlknecht G, Maslak K, Ribba B, Sela M, Possinger K, Yarden Y. 2011. Simultaneous inhibition of estrogen receptor and the HER2 pathway in breast cancer: effects of HER2 abundance. Transl Oncol 4: 293-300. doi:10.1593/tlo.11127

Femino AM, Fay FS, Fogarty K, Singer RH. 1998. Visualization of single RNA transcripts in situ. Science 280: 585-590. doi:10.1126/sci ence.280.5363.585 
Ke R, Mignardi M, Pacureanu A, Svedlund J, Botling J, Wählby C Nilsson M. 2013. In situ sequencing for RNA analysis in preserved tissue and cells. Nat Methods 10: 857-860. doi:10.1038/nmeth .2563

Krzywkowski T, Nilsson M. 2017. Fidelity of RNA templated endjoining by chlorella virus DNA ligase and a novel iLock assay with improved direct RNA detection accuracy. Nucleic Acids Res 45: e161. doi:10.1093/nar/gkx708

Larsson C, Grundberg I, Söderberg O, Nilsson M. 2010. In situ detection and genotyping of individual mRNA molecules. Nat Methods 7: 395-397. doi:10.1038/nmeth.1448

Lee JH, Daugharthy ER, Scheiman J, Kalhor R, Yang JL, Ferrante TC, Terry R, Jeanty SSF, Li C, Amamoto R, et al. 2014. Highly multiplexed subcellular RNA sequencing in situ. Science 343: 13601363. doi:10.1126/science.1250212

Lein E, Borm LE, Linnarsson S. 2017. The promise of spatial transcriptomics for neuroscience in the era of molecular cell typing. Science 358: 64-69. doi:10.1126/science.aan6827

Liu Y, Le P, Lim SJ, Ma L, Sarkar S, Han Z, Murphy SJ, Kosari F, Vasmatzis G, Cheville JC, et al. 2018. Enhanced mRNA FISH with compact quantum dots. Nat Commun 9: 4461. doi:10 .1038/s41467-018-06740-x

Lohman GJS, Zhang Y, Zhelkovsky AM, Cantor EJ, Evans TC. 2014. Efficient DNA ligation in DNA-RNA hybrid helices by Chlorella virus DNA ligase. Nucleic Acids Res 42: 1831-1844. doi:10.1093/ nar/gkt1032

Monici M. 2005. Cell and tissue autofluorescence research and diagnostic applications. Biotechnol Annu Rev 11: 227-256. doi:10 .1016/S1387-2656(05)11007-2

Neumann M, Gabel D. 2002. Simple method for reduction of autofluorescence in fluorescence microscopy. $J$ Histochem Cytochem 50: 437-439. doi:10.1177/002215540205000315

Nilsson M, Malmgren H, Samiotaki M, Kwiatkowski M, Chowdhary BP, Landegren U. 1994. Padlock probes: circularizing oligonucleotides for localized DNA detection. Science 265: 2085-2088. doi:10 $.1126 /$ science. 7522346

Richardson DS, Lichtman JW. 2015. Clarifying tissue clearing. Cell 162: 246-257. doi:10.1016/j.cell.2015.06.067
Schneider N, Meier M. 2017. Efficient in situ detection of mRNAs using the Chlorella virus DNA ligase for padlock probe ligation. RNA 23: 250-256. doi:10.1261/rna.057836.116

Schnell SA, Staines WA, Wessendorf MW. 1999. Reduction of lipofuscin-like autofluorescence in fluorescently labeled tissue. J Histochem Cytochem 47: 719-730. doi:10.1177/00221554990 4700601

Shah S, Lubeck E, Schwarzkopf M, He T-F, Greenbaum A, Sohn CH, Lignell A, Choi HMT, Gradinaru V, Pierce NA, et al. 2016a. Single-molecule RNA detection at depth by hybridization chain reaction and tissue hydrogel embedding and clearing. Development 143: 2862-2867. doi:10.1242/dev.138560

Shah S, Lubeck E, Zhou W, Cai L. 2016b. In situ transcription profiling of single cells reveals spatial organization of cells in the mouse hippocampus. Neuron 92: 342-357. doi:10.1016/j.neuron.2016.10 .001

Soares RJ, Maglieri G, Gutschner T, Diederichs S, Lund AH, Nielsen BS, Holmstrøm K. 2017. Evaluation of fluorescence in situ hybridization techniques to study long non-coding RNA expression in cultured cells. Nucleic Acids Res 46: e4. doi:10.1093/ nar/gkx946

Van de Lest CHA, Versteeg EMM, Veerkamp JH, Van Kuppevelt TH. 1995. Elimination of autofluorescence in immunofluorescence microscopy with digital image processing. J Histochem Cytochem 43: 727-730. doi:10.1177/43.7.7608528

Volpi CC, Gualeni AV, Pietrantonio F, Vaccher E, Carbone A, Gloghini A. 2018. Bright-field in situ hybridization detects gene alterations and viral infections useful for personalized management of cancer patients. Expert Rev Mol Diagn 18: 259-277. doi:10 $.1080 / 14737159.2018 .1440210$

Wang F, Flanagan J, Su N, Wang LC, Bui S, Nielson A, Wu X, Vo HT, Ma XJ, Luo Y. 2012. RNAscope: a novel in situ RNA analysis platform for formalin-fixed, paraffin-embedded tissues. J Mol Diagn 14: 22-29. doi:10.1016/j.jmoldx.2011.08.002

Wu C, Simonetti M, Rossell C, Mignardi M, Mirzazadeh R, Annaratone L, Marchiò C, Sapino A, Bienko M, Crosetto N, et al. 2018. RollFISH achieves robust quantification of single-molecule RNA biomarkers in paraffin-embedded tumor tissue samples. Commun Biol 1: 209. doi:10.1038/s42003-018-0218-0 

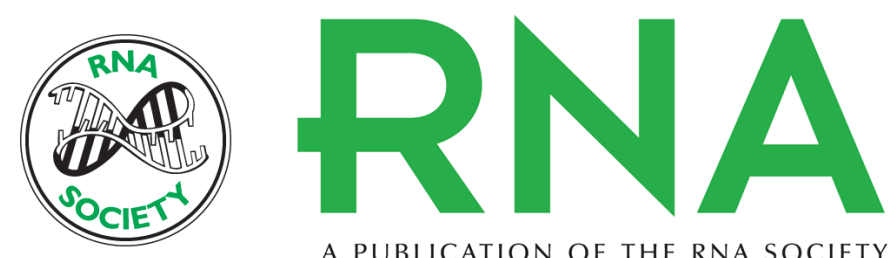

A PUBLICATION OF THE RNA SOCIETY

\section{Single molecule chromogenic in situ hybridization assay for RNA visualization in fixed cells and tissues}

Meng Jiang, Ling Liu, Chengye Hong, et al.

RNA 2019 25: 1038-1046 originally published online May 7, 2019

Access the most recent version at doi:10.1261/rna.070599.119

\section{Supplemental http://rnajournal.cshlp.org/content/suppl/2019/05/07/rna.070599.119.DC1 Material}

References This article cites 29 articles, 7 of which can be accessed free at: http://rnajournal.cshlp.org/content/25/8/1038.full.html\#ref-list-1

Creative This article is distributed exclusively by the RNA Society for the first 12 months after the Commons License full-issue publication date (see http://rnajournal.cshlp.org/site/misc/terms.xhtml). After 12 months, it is available under a Creative Commons License (Attribution-NonCommercial 4.0 International), as described at http://creativecommons.org/licenses/by-nc/4.0/. Email Alerting
Service 